\title{
PRINCIPAL-AGENT APPROACH TO ENVIRONMENTAL IMPROVEMENTS POLICIES
}

\author{
WOJCIECH SZATZSCHNEIDER \\ Actuarial Sciences School, Universidad Anáhuac México Norte \\ Av. U. Anáhuac, Col. Lomas Anáhuac, México \\ E-mail:wojciech@anahuac.mx \\ TERESA KWIATKOWSKA \\ Philosophy Department, U. Autónoma Metropolitana-Iztapalapa \\ San Rafael Atlixco 186, Col. Vicentina, México \\ E-mail: tkwiatkowska@yahoo.com
}

\begin{abstract}
Successful solution to any environmental problem implies working with Knightian uncertainty that explicitly deals with decision making under conditions of unstructured randomness. A 'wild' type of randomness that we will never discern due to its unstable properties makes the assignment of corresponding probabilities impossible. For that reason, the consideration of general economical factors within cost/benefit analysis must fail. So, instead of governmental intervention and a cup and trade scheme, we propose a direct financial market of certificates on environmental improvements. The approach is based on the Principal-Agent method. In this study we analyze the problem of one agent only, and take as example deforestation issues.

In the analytical part we give examples how these certificates could be defined. We solve some optimization problems in well established stochastic models. The approximate knowledge which actions are environmentally friendly allows us to design good environmental certificates.
\end{abstract}

1. Introduction. The Kyoto Protocol, the most popular agenda for reducing pollution by trading carbon quotas, has been raising doubts whether it in fact cleans the environment or just shifts polluting gasses around. It seems that the permits-to-pollute approach takes the worst of the poles: strong governmental intervention (with all the bureaucracy behind), and wild free market. History and the recent financial crisis prove that each one fails over the long term, and the mixture of both cannot lead to positive results. In his

2010 Mathematics Subject Classification: 65K10, 60J60, 62P12.

Key words and phrases: Principal-Agent, environmental improvements, stochastic optimal control.

The paper is in final form and no version of it will be published elsewhere. 
recent book R. Spencer wrote "Unfortunately, carbon taxes international income redistribution schemes like Kyoto protocol destroy existing wealth and prevent the creation of new wealth. These punitive policies then become economically counterproductive".

Many policy-related research develop increasingly complex models that generate a never-ending debate over their applicability, since models entries can hardly be observed or estimated.

Market has been unable to put a price on biological resources. The attempt to price environment by so called contingent valuation resulted impractical, cf. Bateman, Turner (1992).The main idea was to ask how much one would pay for specific environmental goods. This method was extremely unstable with respect to the order of questions.

Any comprehensive approach to conservation of the entire biological diversity requires a strategy that goes beyond economic cost-benefit valuation. Philip E. Graves wrote: "To the extent that we value public goods, we also realize that getting extra income to buy them will accomplish nothing. There is no market to which we can buy, say, reduced CO2 level or endangered species preservation" (2001).

We assume that the environmental deterioration could be stopped o diminished by "good environmental certificates" 1 only.

It is important to stress that we do not pretend to price environment by endowing it with market value. What we propose is the direct market of certificates on environmental improvements, when high reliability measurement of actual state could be ensured: for example the number of wind turbines, trees or (better) biomass. The "conditional carrot" approach using the "Principal-Agent" methodology might be the only way to deal with the most serious environmental crisis.

On the whole, the Principal-Agent method (Nature being the Principal represented by a financial institution) aims at creating new investment opportunities. Agent could be anyone who buys certificates. In reforestation case, these certificates could be given to the inhabitants of a community free of charge. Certificated can be traded. Although in this study we consider one agent only, the method can efficiently stimulate the cooperation between agents when certificates cover the entities like industries, countries or even blocks of them. It could also promote transfer of technologies while permits to pollute do not. The proposed method offers transparency in handling conservation funds that will be created from taxes, or voluntary contributions.

Free Market Environmental (FME) stresses the property rights. See Anderson and Huggins (2008). Our approach pretends to fill its huge gap in cases when property rights can not be ascertained in any direct way (air pollution or in multiple forest dilemmas in developing countries when establishment of property rights is difficult if not impossible).

Our modeling will concern trees. It is just the simplification of more complex models and can be applied for many other environmental topics. Without substantial changes it could be energy production for a given level of pollution. Pollution problems can be treated in a different way, however (certificates of improvement could be decreasing func-

\footnotetext{
${ }^{1}$ Good certificate is meant to stimulate and encourage positive environmental actions like reforestation, restoration, conservation of biodiversity or reduction of pollutants. These certificates can be freely bought by all interested agents.
} 
tions of emissions). Our models are just examples of how certificates can be handled and not to take them as a rule for modeling.

2. The models. To explain how relevant optimization problems for good environmental certificates could be handled, we will introduce two models (diffusions). In the first model we are able to solve the problem completely in the case of linear certificates. In the second model, using dual technique, we represent the optimal agent's action (under additional assumption and in the case of logarithmic award) in terms of the corresponding Lagrange multiplier.

To find it, one has to solve the equation involving the expectation of complicated but in a sense elementary functionals of Brownian Motion, at the moment we offer a semi-closed solution.

We provide "soft" theoretical justification of both. Further analysis of their real applicability and calibration depends on experience. In our setting, to start the project, we do not need precise models! 2 "Good" certificates would contribute positively to the environment in any circumstances!

3. General problem. Consider a static local goal with the horizon $T$. The general problem is the following: given fund $S$, emit (Principal) certificate $F$ that after the optimal agent's action $u(F)$, generates

$$
\max _{F} E\left\{\int_{0}^{T} f(s) X_{s}^{u(F)} d s\right\}
$$

where $X_{s}^{u}=X_{s}^{u(F)}$ represents the number of trees at time $s$ in a given area.

More formally: Let $u$ be the agent's action adapted to the Brownian filtration that modifies the dynamic of the corresponding process $X_{s}^{u}$, by adding

$$
u=u(F, s),
$$

where $F\left(X_{s}^{u}, s \leq T\right)$ is the agent's award at time $T$. Here $0 \leq F \leq S$, and $S$ is the corresponding fund. Let

$$
J_{1}(F, u)=E\left(F\left(X_{s}^{u}, s \leq T\right)-\text { cost }\right), \quad J_{2}(F, u)=E\left(\int_{0}^{T} f(s) X_{s}^{u(F)} d s\right) .
$$

The problem consists in the maximization of two functionals stemming from:

1 Agent's optimization problem,

$$
\sup _{u} J_{1}(F, u)=J_{1}\left(F^{*}, u^{*}\right) .
$$

$J_{1}$ becomes the agent's net monetary award.

2 Principal's optimization problem that emits certificates $F$,

$$
\sup _{F} J_{2}(F, u(F))=J_{2}\left(F^{*}, u^{*}\right) .
$$

\footnotetext{
${ }^{2}$ This is the main reason that we do not venture to analyze more complex economic models.
} 
In our first model we will consider $f(s) \equiv 1$ only. Decreasing $f$ would, however, be more suitable.

The Principal representing the Nature is here the financial institution in charge of the management of the fund. The Principal issues certificates to agents.

We assume that $X(s)$ can be monitored with desirable reliability.

Awards are limited by funds. There are two general possibilities to work with bounded awards:

1. Set bounds on unbounded models.

2. Consider bounded models.

It seems that the second choice is easier to analyze. In the first choice it is unclear how models should be handled after hitting the corresponding barrier and, moreover, after the optimal agents's action the hitting time usually doesn't have explicit probability law. Moreover in several specific applications like reforestation issues, models are bounded by respective capacities. Therefore bounded models are more appropriate. In biological systems the Pearl-Verhulst model defined by $d V(t)=V(t)(1-V(t)) d t+V(t) d W(t)$ represents the growth with saturation. However we need models with absolute saturation (bounded). Our examples meet these requirements.

We shall assume here agent's linear utility and thus without losing the general approach one certificate sold. For practical applications certificates should be traded producing possibly the concentration of capital in the hands of powerful individuals able to stop environmental degradation.

As an advance of more general analysis we will study two models with some restrictions on awards and optimal agent's actions. In both models we shall work with more continuous time models as an approximation of "discrete time reality."

4. Model 1. The number of trees in a given area is represented by the diffusion process $X(t)$ defined by:

$$
d X(t)=f[X(t)(k-X(t))] d W(t)+(u(t)-\beta)(k-X(t)) d t,
$$

with $W(t)$ Brownian Motion, $X(0)=x, 0<x<k, k$ is the capacity, and $f$ is any function ensuring existence and uniqueness of the solution, cf. Revuz, Yor (1999); and $f(0)=0$. For example $f(x)=|x|^{\alpha}, \alpha \geq \frac{1}{2}$. We will impose conditions such that the optimal agent action $u^{*}(t) \geq \beta$ and it will be deterministic, therefore our comment about the existence and uniqueness holds. In this case $0 \leq X(t) \leq k$. We will see that solutions of corresponding optimization problems will be $f$ free.

To simplify the notation we shall set $k=1$. We use this parametrization factorizing $u(s)$ and $\beta$, to ensure the absolute saturation. There is some intuitive motivation of this model. Large $X$ means no big environmental problems and consequently, lower deforestation rate, while interpreting $u(s)$ as an effort, this produces smaller changes in the drift. Another pattern will be considered in model 2 . Here we will consider linear awards only.

It is convenient to write the award as

$$
-A \int_{0}^{T}(1-X(s)) d s-\gamma(1-X(T))+S,
$$


where $A, \gamma, S \geq 0$, and the agent's cost is $\delta \int_{0}^{T} u^{2}(s)(1-X(s)) d s$. This cost can be seen as a compromise between both: quadratic (of effort) and linear (of actual state). The cost is smaller for larger $X$ (reforestation is cheaper). For simplicity sake we set $\delta=1$. We have to choose optimal $A$ and $\gamma$ such that after the optimal agent's action $E\left(\int_{0}^{T} X_{s}^{u^{*}(A, \gamma)} d s\right)$ would be maximized.

THEOREM 4.1. For linear certificates and under the condition that optimal agent's effort $u$ is bounded for any $T>0$, the optimal linear certificate is: take $A$ as large as possible with the following restrictions:

$$
A \leq \beta^{2}, \quad T A+\gamma \leq S, \quad \gamma-2 \beta \geq 0
$$

Proof. The Bellman approach leads to the following equation for the value function:

$$
F_{t}(x, t)+\left(\frac{F_{x}^{2}(x, t)}{4}-\beta F_{x}(x, t)+A+\frac{1}{2}[f(x(1-x))] F_{x x}(x, t)\right)(1-x)=0 .
$$

We will find the solution of the form $F(x, t)=h(t)(1-x)$ and so it will be $f$ free. We get the following equation for $h(t)$ :

$$
h^{\prime}(t)+\left(\frac{1}{2} h(t)+\beta\right)^{2}-\beta^{2}+A=0 .
$$

Setting $h(t)=4 H(t)-2 \beta$ we obtain the trivial Riccati equation

$$
H^{\prime}(t)+H^{2}(t)=\frac{\beta^{2}-A}{4} .
$$

In our setting it is desirable to ensure the existence of bounded agent's optimal action (independently of $T$ ). This fact translates easily into $\beta^{2} \geq A$. So we have to solve the problem with the following constraints:

1. $A \leq \beta^{2}$

2. $T A+\gamma \leq S$

3. $\gamma-2 \beta \geq 0$

The second and third conditions result from the fact that the total award in any case must be positive and, respectively, to ensure that $u^{*}(t) \geq \beta$ for $0 \leq t \leq T$. Writing

$$
H(t)=\frac{\varphi^{\prime}(t)}{\varphi(t)}, \quad \varphi(0)=1
$$

we have

$$
\frac{\varphi^{\prime \prime}(t)}{\varphi(t)}=\frac{\beta^{2}-A}{4},
$$

and if $\beta^{2}>A$ then $\varphi(t)=B e^{\alpha t}+(1-B) e^{-\alpha t}$ with

$$
\alpha=\frac{\sqrt{\beta^{2}-A}}{2}, \quad B=\frac{(4 \alpha+2 \beta-\gamma) e^{-\alpha T}}{(4 \alpha-2 \beta+\gamma) e^{\alpha T}+(4 \alpha+2 \beta-\gamma) e^{-\alpha T}},
$$

while if $\beta^{2}=A$, then the solution for $\varphi$ is linear.

Now optimal $u^{*}(t)=-h(t) / 2 \geq \beta$. Elementary calculations show that $E\left(\int_{0}^{\alpha} X(s) d s\right)$ will be maximized if $\int_{0}^{t} \varphi^{2}(s) d s$ would take the minimal possible value. If $A<\beta^{2}$, this 
means the minimization with respect to $A$ and $\gamma$ of

$$
\frac{1}{2 \alpha}\left\{B^{2}\left(e^{2 \alpha T}-1\right)+(1-B)^{2}\left(1-e^{-2 \alpha T}\right)\right\}+2 B(1-B) T .
$$

After some elementary work we obtain that the solution is: Set $A$ as large as possible.

5. Model 2. The second model is defined by

$$
d X(t)=X(t)(k-X(t)) d W(t)+(\delta u(t)-\beta) X(t)(k-X(t)) d t
$$

As before we set $k=1, \delta=1$, and for example $\beta=\frac{1}{2}, X(0)=\frac{1}{2}$. We are able to obtain the semi-closed solution of agent's optimization problem in the case of an award $F(X(T))$ with special choice of $F$, and special form of agent's action.

In this model the deforestation and effort effect produce larger changes of drift for middle sizes of $X(t)$.

Assume that the agent cost is $\int_{0}^{T} \frac{u^{2}(s)}{2} X(s)(1-X(s) d s$. The motivation is similar to given in the previous model.

Our solution will be based on heavy use of Girsanov's theorem that will change the law of corresponding process to the one of the auxiliary model:

$$
Y(t)=\frac{1}{1+e^{-W(t)}} .
$$

By Itô's Lemma:

$$
d Y(t)=Y(t)(1-Y(t)) d W(t)+\left[\frac{1}{2} Y(t)(1-Y(t))-Y^{2}(t)(1-Y(t))\right] d t .
$$

Set $Z(t)=e^{\frac{1}{2} W(t)-\frac{1}{8} t}$. Now

$$
M(t)=X(t) Z(t)-\int_{0}^{t} u(s) Z(s) X(s)(1-X(s)) d s
$$

is clearly a martingale, $M(0)=x=\frac{1}{2}$. Therefore $\forall z, E(-z M(T)+z x)=0$.

Assume that the agent acts in locally optimal way. It means that at time $s$ chooses the adopted action $u(s)$ that maximizes instantaneous drift minus cost if written as $H(u(s), W(s)) \cdot F(X(s))$; taking $X(s)$ as a constant.

The set of strategies that depend actually on $W(s)$ only, we shall call $\mathcal{U}$. Because $X(s)$ depends on $u(s)$, there is no reason that this procedure will produce globally optimal strategy.

Using standard dual method, our goal is to maximize with respect to $u(s) \in \mathcal{U}$

$$
\begin{aligned}
E(F(X(T))-c o s t)= & E\left(F(X(T))-\int_{0}^{T} \frac{u^{2}(s)}{2} X(s)(1-X(s)) d s\right) \\
= & E\left(\left(F(X(T))-z X(T) Z(T)-\int_{0}^{T} \frac{u^{2}(s)}{2} X(s)(1-X(s)) d s\right.\right. \\
& \left.+z \int_{0}^{T} u(s) Z(s) X(s)(1-X(s)) d s+z x\right) .
\end{aligned}
$$

To make calculations more explicit we choose:

$$
F(x)=\ln (x+B), \quad B>1 .
$$


THEOREM 5.1. The optimal agent's locally optimal effort $u(t)=\check{z} Z(t)$ and optimal

$$
X_{T}=\left\{\begin{array}{cl}
\frac{1}{\bar{z} Z(t)}-B & \text { if } \frac{1}{\bar{z} Z(t)}-B \in(0,1), \\
0 & \text { if } \frac{1}{\bar{z} Z(t)}-B \leq 0, \\
1 & \text { if } \frac{1}{\bar{z} Z(t)}-B \geq 1 .
\end{array}\right.
$$

$\check{z}$ is the Lagrange multiplier and can be calculated from equation (1) that involves expectation (2) of functionals of Brownian motion given by (3) and (4).

Proof. Standard approach gives formulas for $u(t)$ and $X(T)$. The Lagrange multiplier $\check{z}$ is such that

$$
E\left(Z(T) X(T)-\check{z} \int_{0}^{T} Z^{2}(s)(1-X(s)) X(s) d s\right)=\frac{1}{2}
$$

The only problem to calculate $\check{z}$ is to calculate

$$
E \int_{0}^{T} Z^{2} X(s)(1-X(s)) d s=\tilde{E} \int_{0}^{T} e^{\frac{1}{4} s} X(s)(1-X(s)) d s
$$

where under the " $"$ law

$$
d X(t)=X(t)(1-X(t)) d W(t)+\left[\check{z} e^{W(t)+\frac{1}{2} t}+\frac{1}{2}\right] X(t)(1-X(t)) d t .
$$

Firstly we take away the term $\check{z} e^{W(t)+\frac{1}{2} t} X(t)(1-X(t))$ using Girsanov's theorem:

$$
\tilde{E}\left(\int_{0}^{T} e^{\frac{1}{4} s} X(s)(1-X(s)) d s\right)=E^{*}\left(\int_{0}^{T} e^{\frac{1}{4} s} X(s)(1-X(s)) d s \cdot \mathcal{E}\left(\int_{0}^{\cdot} h(s) d W^{*}(s)\right)_{T}\right)
$$

where

$$
h(s)=\frac{e^{W^{*}(s)+\frac{1}{2} s}}{\frac{1}{\check{z}}+\int_{0}^{s} e^{W^{*}(u)+\frac{1}{2} u} d u} .
$$

We use the standard notation: for given martingale $M, \mathcal{E}(M)_{T}$ means $e^{M_{T}-\frac{1}{2}<M, M>_{T}}$.

This change of measure needs justification. We leave it to the appendix.

Under $P^{*}$ law

$$
d X(t)=X(t)(1-X(t)) d W^{*}(t)+\frac{1}{2} X(t)(1-X(t)) d t
$$

which is much closer to our auxiliary process.

Secondly and lastly we use once again Girsanov's theorem to express the expectation (2) as

$E\left\{\int_{0}^{T} e^{\frac{1}{4} s} X(s)(1-X(s)) d s \cdot \mathcal{E}\left(\int_{0}^{\cdot} \tilde{h}(s) d\left(W(s)-\int_{0}^{s} X(u) d u\right)\right)_{T} \cdot \mathcal{E}\left(\int_{0}^{\cdot} X(s) d W(s)\right)_{T}\right\}$

where $\tilde{h}(s)$ is $h(s)$ but with $W^{*}(s)$ replaced by $W(s)-\int_{0}^{s} X(u) d u$. Under this new law

$$
X(s)=\frac{1}{e^{-W(s)}+1}
$$


Making the corresponding replacement for $W^{*}(s)$, we need to calculate:

$$
\begin{gathered}
E\left\{\left(\int_{0}^{T} e^{W(s)+\frac{1}{4} s}\left(e^{W(s)}+1\right)^{-2} d s\right)\right. \\
\times \exp \left[-\int_{0}^{T} h(s)+\left(e^{W(s)}+1\right)^{-1} d W(s)\right] \\
\left.\times \exp \left[-\int_{0}^{T} h(s)\left(1+\frac{1}{2} h(s)\right)+\left(2 e^{W(s)}+2\right)^{-1} d s\right]\right\},
\end{gathered}
$$

where

$$
h(s)=\frac{\check{z} \exp \left[-W(s)+\frac{1}{2} s-\int_{0}^{s}\left(e^{W(u)}+1\right)^{-1} d u\right]}{1+\check{z} \int_{0}^{s} \exp \left[-W(u)+\frac{1}{2} u-\int_{0}^{u}\left(e^{W(\tau)}+1\right)^{-1} d \tau\right] d u} .
$$

This formula jointly with (1) represents what we called previously semi-closed solution.

\section{Conclusions.}

1. This study extends our previous ones (2003),(2004). See also D'Amato and Franckx (2003). In their study the authors considered the case when "The private agent can allocate its effort to environmental protection or to its core task". In this case the role of proper choice of parameters, unlike in our case, can not be avoided.

2. The course of actions we propose is a trade-off between environmental concern and economic benefits for those involved; rewarding positive environmental actions like and planting trees (reforestation) or polluting less and, more importantly, by stimulating transfer of new technologies.

3. Certificates that embrace larger regions could automatically generate these necessary transfers.

4. Different optimization problems could be formulated and solved for certificates of the form $S-\int_{0}^{t} F(X(s)+Y(S)) d s$ for some convex function $F$. Here $X(s)$ and $Y(s)$ represent pollution level in two regions. Allowing agent 1 living in region 1 to make improvements in region 2 can be called fusion and lead directly to transfer of technologies beyond well studied collusive or Nash equilibrium. This approach is now in development in our studies with promising results.

5. We hardly know the social cost of pollution or deforestation. In first applications one can use any good environmental certificates and do not worry about their optimalities in specific models behind.

7. Appendix. Without loss of generality we may assume $\check{z}=1$. To justify the second change of measure we need to prove that

$$
H_{T}=\exp \left[\int_{0}^{T} V(s) d W(s)-\frac{1}{2} \int_{0}^{T} V^{2}(s) d s\right]
$$

is a true martingale and not only a local martingale. Here

$$
V(s)=\frac{e^{W(s)}+\frac{1}{2} s}{1+\int_{0}^{s} e^{W(u)+\frac{1}{2} u} d u}
$$


and satisfies

$$
d V(t)=V(t)(1-V(t) d t+V(t) d W(t) .
$$

The law of this process we will call $P$. The best way to prove that $H(t)$ is true martingale is through some equivalences of laws of processes on $\mathcal{F}_{T}^{V}, \forall T$.

Note that $V(t)$ is the Pearl-Verhulst model.

Let under the law $Q, d V(t)=V(t) d t+V(t) d W(t)$. The law $Q$ is equivalent to the law $Q_{1}$. Under $Q_{1}, V(t)=e^{W(t)-\frac{1}{2} t}$.

Now, under $Q_{1}$

$Z_{T}=\exp \left[-\int_{0}^{T} V(s) d W(s)-\frac{1}{2} \int_{0}^{T} V^{2}(s) d s\right]=\exp \left[-e^{W(T)-\frac{1}{2} T}+1-\frac{1}{2} \int_{0}^{T} e^{2 W(s)-s} d s\right]$.

$Z_{T}$ is clearly true martingale and by Girsanov's theorem changes the law $Q_{1}$ into $\tilde{Q}$ such that under $\tilde{Q}$,

$$
d V(t)=-V^{2}(t) d t+V(t) d W(t)
$$

Moreover $\tilde{Z_{T}}>0$, under $\tilde{Q}$ measure.

Therefore $Q \sim Q_{1} \sim \tilde{Q} \sim P$ on $\mathcal{F}_{T}$, and we have proved that $Z_{T}$ is a $P$ martingale. The last equivalence is obvious.

\section{References}

[1] T. L. Anderson and L. E. Huggins, Greener Than Thou. Are You Really an Environmentalist?, Hoover Institution Press, Stanford University, 2008.

[2] I. Bateman and K. Turner, Evaluation of the environment: The contingent valuation method, CSERGE Working Paper GEC, 92-18.

[3] A. D'Amato and and L. Franckx, Environmental policy as a multi-task principal-agent problem, Energy, Transport and Environmental Working papers, Faculty of Economics, Katholieke Universiteit Leuven 12 (2003).

[4] P. E. Graves, Valuing public goods, Working paper, University of Colorado, 2001.

[5] D. Revuz and M. Yor, Continuous Martingales and Brownian Motion, third edition, Springer, 1999.

[6] R. W. Spencer, Climate Confusion. How global warming hysteria leads to bad science, pandering politicians and misguided policies that hurt the poor, Encounter Brooks, New York, London, 2008.

[7] W. Szatzschneider, M. Jeanblanc and T. Kwiatkowska, Environment $\&$ financial markets, Springer Lecture Notes in Computer Sciences 3039 (2004), 787-794.

[8] W. Szatzschneider and T. Kwiatkowska, Environment \& financial markets, ARCH Electronic Journal of the Society of Actuaries, January (2003). 
